\title{
Mortality of silkworms due to air pollution: environmental indicators induced by ecological stress
}

\author{
E. Kamilova ${ }^{1} \&$ B. Tsarev ${ }^{2}$ \\ ${ }^{1}$ Institute of Genetics and Plant Experimental Biology, \\ Academy of Sciences of Uzbekistan, Uzbekistan \\ ${ }^{2}$ National University of Uzbekistan, Uzbekistan
}

\begin{abstract}
The present investigation is the first attempt in determining stress-induced changes in a soil-plant-insect ecosystem in an aluminum works zone to create a mathematical model satisfactorily describing the process of silkworm destruction due to the harmful emissions of an aluminum works and to develop an algorithm for rating the ecological risk and measures of its prevention. The major objective of this research was to create scientifically based recommendations for predicting the level of damage to the surrounding ecosystems by the aluminum works and to take adequate measures to protect the Great Silk Road. The investigations have been conducted by the evaluation of toxic metal accumulation in the ecosystem components with the distance limit of the wind rose exposure. Concentrations of manganese, iron, cobalt, zinc, cadmium, chromium, rubidium, selenium, scandium antimony, strontium, lithium, lead, mercury, silver, aluminum, beryllium, molybdenum, nickel, tin and copper were detected by the atomic absorption spectrophotometry method. The fluoride content was determined by the alizarinechelatometry photometry method. The theoretical basis of the algorithm was the theory of the survival of organisms under conditions of stress based on Strehler-Mildvan's model. The data were processed by the ANOVA computer statistic tests. The real ecological process was successfully described by a forecast made on the basis of the obtained mathematical model. As a result, we have obtained the findings regarding the accumulation of zinc, nickel, mercury, antimony, etc. in the soils, silkworm excrements and the cocoons near the works. We have also obtained the threshold concentrations of the above mentioned elements in the ecosystem components leading to mortality of the silkworms and cocoons.
\end{abstract}

Keywords: ecosystems modelling, silkworms, ecological risks, aluminum works. 


\section{Introduction}

In recent years many researchers have attempted to quantify the impact of synergetic influence and the accumulation of fluorine and toxic metals in contaminated sites on human health and the environment (Baars [1], Cerklewski [2], Dabeka [3], Davydova [4], Elsair [5]). However, the authors of the present paper have not found references describing fluorine, fluorides and toxic metal influence on the growth and development of Mulberry (Morus alba L.) and silkworms.

The research has been carried out in the aluminum works zone in Surkhandario velayat in the Republic of Uzbekistan. The Tadjiksky aluminum works have been the biggest source of fluorine (F) leaks in Surkhandario velayat in Uzbekistan in recent years, which led to wide dispersal of this toxic pollutant in the Surkhandario valley. The Tadjiksky aluminum works also eject a wide range of toxic metals into the atmosphere. This paper explains the general impact of the air pollution of fluorine and toxic metals on the ecosystems' components.

One of the important tasks of the investigation was to develop new techniques for determining toxic metals in components of the ecosystem using the Atomic Absorption Spectrophotometry method. The paper explains the methodology of sampling and analysis, methods for the ambient soil, plants and animal tissues.

Fluorine destroys the gardens that are situated in the area close to the aluminum works. There is also a high concentration of fluorine in grass and pasture for domestic animals. The consequences of this phenomenon are leading to a reduction in the number of domestic animals in the area surrounding the works and to poor quality of the milk and meat that have become inedible. Fluorine impairs the growth and development of silkworms in the surrounding area and leads to a high rate of their mortality.

In recent years there has been considerable concern about human-induced environmental change in Uzbekistan. High rates of air pollution and extremely high inputs of chemicals have resulted in a build up of fluorides and toxic metals in the soils and water bodies of the region, and sediments deflated from these sources are likely to be highly toxic. In the polluted zone, the accumulation of zinc, nickel, mercury, antimony and a wide range of other toxic metals in the soils, silkworm excrements and the cocoons was revealed.

\section{Materials and methods}

\subsection{Data collection}

For the study of the fluorine effect as a possible cause of silkworm destruction in the zone of emission of fluorine pollution by the aluminum works, investigations have been conducted for the evaluation of fluoride accumulation in the ecosystem components (soil, Mulberry leaves and sticks, apricot-tree leaves and sticks, silkworm caterpillars and excrements of silkworm).

Concentrations of fluoride as a possible cause of silkworm death in the aluminum works zone and the accumulation of fluorides by insect and plant tissue were measured twice during the two-year study period. The first run was 
undertaken in May 1999, and the second one in June 2000. The samples were taken at certain sites in Surkhandario velayat in Uzbekistan. The evaluation of fluoride accumulation was investigated in three tumans (regions) of Uzbekistan with the following distance limits from the zone of fluorine emission (Tadjiksky aluminum works):

1. Saryossio site: $27.5 \mathrm{~km}$ from the aluminum works (the polluted zone, silkworms die).

2. Denov site: $41 \mathrm{~km}$ from the aluminum works (the intermediate zone, destruction of growth (or impairment of growth) and development of silkworms appeared).

3. Djarkurgan site: $134 \mathrm{~km}$ from the aluminum works (the non-polluted, control zone).

The Surkhandario Valley is situated between two mountain ranges. As a result, there is some kind of a wind tunnel along the valley. The airflow transfers harmful emissions from the Tadjiksky aluminum works from Saryossio up to Dzharkurgan.

\subsection{Toxic metals monitoring}

Concentrations of manganese, iron, cobalt, zinc, cadmium, chromium, rubidium, selenium, scandium antimony, strontium, lithium, lead, mercury, silver, aluminum, beryllium, molybdenum, nickel, tin and copper were detected by the Atomic Absorption Spectrophotometry method (Perkin Elmer 3030B). The Environmental Compliance Standard PCS-1 (Pollution Control Standard \#1) was used as the standard for this investigation.

The fluoride content was determined by the alizarinechelatometry photometry method (Leonard [6]).

The data were processed by the ANOVA computer statistic tests.

\section{Results}

\subsection{Mathematical modeling of life and development of the silkworm Bombyx mori}

A larva has five instars (periods when larvae feed on mulberry leaves) and four sleeping periods (when larvae stop feeding and are at rest). Numerical simulations were carried out under the conditions that:

$i-$ number of the stage, $\quad i=1,2, \ldots 9$;

$\mathrm{k}$ - number of the instar, $\quad \mathrm{k}=1,2,3,4,5$;

$\mathrm{n}$ - number of the sleeping period; $\mathrm{n}=1,2,3,4$.

In this case,

$$
k=\operatorname{int}\left[\frac{(i+1)}{2}\right] ; \quad n=\operatorname{int}\left[\frac{i}{2}\right]
$$

Let $\tau$ be measured in days (or $\mathrm{t}$ in hours), $T=\sum \tau_{i}$. 
Let us calculate the accumulation of the larva's weight to the end of the $\mathrm{j}$-th stage. If $\Delta m_{i}$ is equal to the integral change of weight in time applicable to the $\mathrm{i}$-th stage, the weight of the larva at the end of the j-th stage will be:

$$
m_{j}=m_{0}+\sum_{i=1}^{j} \Delta m_{i}, \quad \text { where } \quad \max (j)=9
$$

A change of larva's weight differs in active (instar) and passive stages (the sleeping period). Within the limits of any k-th stage the accumulation of weight goes on because of intensive Bombyx larvae eating a lot of mulberry leaves day by day (except during the "sleeping period") with the velocity proportional to the weight already gained.

$$
\left(\frac{d m}{d t}\right)_{k}=\beta_{k} m, \mathrm{k}=\mathrm{I}, \mathrm{II}, \mathrm{III}, \mathrm{IV}, \mathrm{V} .
$$

Integrating from here gives:

$$
m_{k}(t)=m_{0, k} \exp \left[\beta_{k}\left(t-t_{0}\right)_{k}\right]
$$

where $m_{0, k}$ is the initial weight of a silkworm larva in the k-th stage; $\beta_{k}$ is an empirical coefficient, day ${ }^{-1}$ (or hours ${ }^{-1}$ ). Coefficient $\beta_{k}$ is the specific velocity of growth rate of a larva. $\beta_{k}^{-1}=\tau_{*}$ is a coefficient inverse to $\beta_{k}$ and determines a time interval, equal to the time period during which the weight of a larva increases by e $=2,72$ times.

$$
\beta_{k}=\frac{1}{\tau_{k}} \ln \left(\frac{m_{+}}{m_{0}}\right)_{k}
$$

The analytical formula for estimation of the accumulation of any substances ( $m_{v}$, in grams) for 1000 larvae at the end of an instar is represented as:

$$
m_{v}=\exp \left(A_{v} k+B_{v}\right)
$$

The accumulation of weight for all periods of the larva's life from hatching to spinning of the cocoon is shown in Figure 1. Inside a line (Fig. 1) the death rate of larvae does not exceed $1 \%$ and is caused, mainly, by mechanical damage. Suppose that for a population of larvae the normal distribution for $\beta_{k}$ takes place, it is possible for the optimal range

$$
\beta_{k}=\bar{\beta}_{k} \pm \varepsilon \sigma_{k}
$$

to determine the values of parameters $\varepsilon$ (displacement) and $\sigma$ (average quadratic deviation). At $\varepsilon=0,674$ probability, $\beta_{k}$ (the specific velocity of growth rate of a larva) lies within the indicated range of $50 \%$, at $\varepsilon=1$ the probability is $68 \%$, at $\varepsilon=2$ the probability reaches $95,5 \%$ and at $\varepsilon=3$ it is about $99,95 \%$.

Let $\varepsilon=3$, in this case $\sigma_{k}=\left(\beta_{\max }-\beta_{\min }\right)_{k} / 6$. 


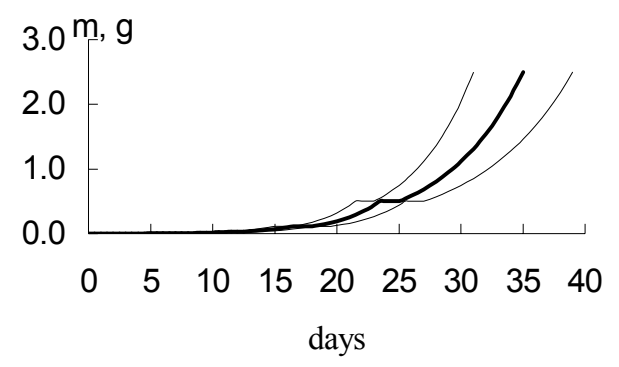

Figure 1: Larvae's weight accumulation.

In Figure 1, the weight gained for the period of life of the silkworm larvae on average and extreme values of $\beta_{k}$ (specific velocity of the growth rate of a larva) presents:

$$
\sigma(k)=\sum_{j=0}^{j=3} a_{j} k^{j} \quad C v(k)=\sum_{j=0}^{j=3} b_{j} k^{j}
$$

As we see, the average quadratic deviation almost does not change, the average is 0,0114 . The coefficient of variation for the fifth instar increases a little. From (4) and (7) the estimation of the maximum duration for each instar follows:

$$
\tau_{\max }=\frac{\tau_{c p}}{1-6 C v} .
$$

Let us consider the specific velocity of the growth rate of a larva as a random quantity for a large number of larvae. Let us record statistical functions, bound with this random variable in the supposition of the validity of a Gauss law.

The density of probability of a normal distribution of the specific velocity of the larva growth rate is expressed by the formula:

$$
f(\beta)=\frac{1}{\sigma \sqrt{2 \pi}} \exp \left\{-\frac{(\beta-\bar{\beta})^{2}}{2 \sigma^{2}}\right\}
$$

The cumulative distribution function $F(\beta)$ is the probability that the random variable $\mathrm{U}$ possesses a value smaller than or equal to $\beta$.

$$
F(\beta)=\int_{-\infty}^{\beta} f(u) d u
$$

The risk function (the intensity of the death rate) is:

$$
h(\beta)=\frac{f(\beta)}{1-F(\beta)}=\frac{f(\beta)}{S(\beta)}
$$

where $S(\beta)$ is the survival function (security).

The cumulative risk function (the integral of the risk function) is (see fig. 2): 


$$
H(\beta)=-\int_{-\infty}^{\beta} h(u) d u=-\operatorname{Ln}(1-F(\beta))=-\operatorname{Ln}(S(\beta)
$$

Under normal conditions of the development of silkworm larvae (and at the average velocity of growth), all of them reach the limiting weight applicable to an instar and pass into the following stage - the sleeping period. Only a very small fraction of underdeveloped larvae die out due to a small velocity of growth $\left(<\beta_{\text {av. }}-3 \sigma\right)$ up until the end of the instar deadline.

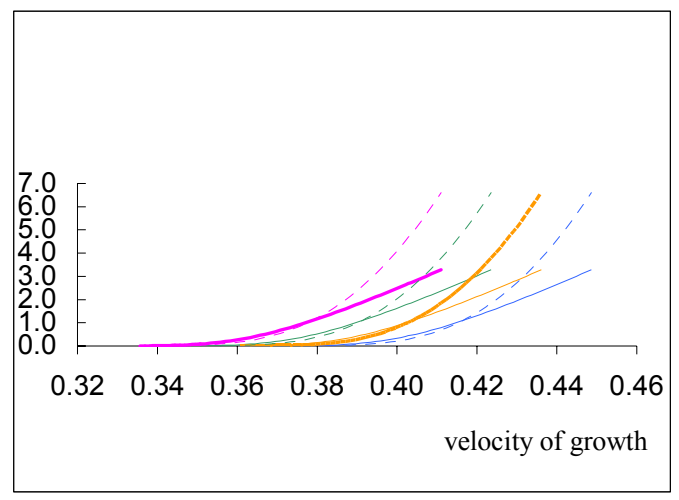

Figure 2: Risks functions and cumulative risk functions.

\subsection{Simulation of ecological stress conditions}

This effect is exhibited in a decrease of the larvae's weight and a decreasing velocity of growth $\beta$ according to the accumulation of toxic elements in larvae during pollution exposure. In the references in which the different theories of death rates are reviewed [7-18], the loss of life expectancy can be shown by a linear function of accumulated pollutants:

$$
\beta_{k}=\bar{\beta}_{k}+g m_{z}=\bar{\beta}_{k}+g C m,
$$

where $\bar{\beta}_{k}$ is the average normal specific velocity of the growth rate of larva, $m_{z}$ is the accumulated weight of pollutants, $m$ is the total accumulated weight of larva, $C$ is the concentration of pollutants in food, $\mathrm{g}$ is the average gradient of a decrease of $\beta_{k}$, the specific velocity of growth rate of larva $\left([\mathrm{g}]=\mathrm{day}^{-1} \mathrm{~g}^{-1}\right)$, and it can only be within the limits:

$$
\left(-\frac{\bar{\beta}}{C m_{0}}<g<0\right)
$$

Substituting (14) into (3) we shall receive:

$$
\frac{d m}{d t}=\bar{\beta} m+g C m^{2} .
$$


The solution of this differential equation at boundary conditions $m(t=0)=m_{0}$ results in the expression:

$$
m=\frac{1}{\left(\frac{1}{m_{0}}+\frac{g C}{\bar{\beta}}\right) \exp (-\bar{\beta} t)-\frac{g C}{\bar{\beta}}},
$$

If we designate

$$
\begin{gathered}
m_{*}=\frac{\bar{\beta}}{g C}, \\
m=\frac{1}{\left(\frac{1}{m_{0}}+\frac{1}{m_{*}}\right) \exp (-\bar{\beta} t)-\frac{1}{m_{*}}} .
\end{gathered}
$$

At concentration $\mathrm{C}=0$ equations (17) and (19) change to (4).

Let us consider equations (7) and (14) jointly:

$$
g=\frac{\varepsilon \sigma}{C m}
$$

If in a time equal to the period of instar $\tau$, a larva accumulates weight $\mathrm{m}$, and, if the accumulation of this weight took the maximum permissible duration of the period causing a decrease of vital powers (14) because of the presence of toxic pollutants in food, (7) and (14) can be written as:

$$
\left\{\begin{array}{l}
\beta=\bar{\beta}+\varepsilon_{\Pi} \sigma \\
\beta=\bar{\beta}+g C_{\Pi} m
\end{array},\right.
$$

where $\varepsilon_{\Pi}$ and $\mathrm{LD}_{100}$ are limiting values of the coefficients of displacement and concentration, respectively. $\mathrm{LD}_{100}$ is the limiting value of concentration (a lethal dose of toxic element exposure that leads to the death of $100 \%$ larvae, $\mathrm{mg} / \mathrm{kg}$ ). Thus, during the maximum duration of an instar only a small part of a larvae population gains the weight necessary to reach the following stage - the sleeping period. This condition, apparently, corresponds to $\varepsilon_{\Pi}=-6$.

For this condition:

$$
\mathrm{LD}_{100}=\frac{\varepsilon_{\Pi} \sigma}{g m},
$$

makes it possible to determine the maximum permissible concentration by the average accumulated weight for any instar, if the value of the average gradient of decreasing $\beta_{k}$ and the specific velocity of the growth rate of larva $([\mathrm{g}]$ $=$ day $^{-1} \mathrm{~g}^{-1}$ ) is known, and depends on the biological role of the polluting chemicals.

From (22) we receive:

$$
\left(\frac{\varepsilon_{G} \sigma}{\mathrm{LD} 100 m}<g<0\right)
$$


Let us enter a parameter of relative concentration of the toxic element $\delta=\mathrm{C} /$ $\mathrm{LD}_{100}$, find from (22) with gradients $\mathrm{g}$ and inserting it into equation (17) to calculate the limiting weight $\mathrm{m}$ depending on relative concentrations of toxic elements and time:

$$
m(\delta, t)=m_{0}\left[\left(1+\frac{\varepsilon_{\Pi} \sigma}{\bar{\beta}} \cdot \delta\right) \cdot \exp (\beta t)-\frac{\varepsilon_{\Pi} \sigma}{\bar{\beta}} \cdot \delta\right] ; 0<\mathrm{t}<\mathrm{t}_{\text {мах }}
$$

Let us find time $t_{\max }$, necessary for the accumulation of the limiting weight by the larva, before transferring to the next stage of life - the sleeping period, using equation (4) for the first instar under the normal conditions of life $\left(\mathrm{t}_{\mathrm{n}}\right)$ and under ecological stress conditions $(23)(0 \leq \delta \leq 1)$.

$$
m_{0} \exp \left(\bar{\beta} t_{H}\right)=m\left(\delta, t_{\max }\right) \Rightarrow t_{\max }=\frac{1}{\bar{\beta}} \cdot \ln \left[\frac{\bar{\beta} \exp \left(\bar{\beta} t_{H}\right)+\varepsilon_{\Pi} \sigma \delta}{\bar{\beta}+\varepsilon_{\Pi} \sigma \delta}\right]
$$

At $\delta=0$ we have $\mathrm{t}_{\max }=\mathrm{t}_{\mathrm{n}}$. At $\delta=1$ and $\varepsilon_{\Pi}=-6$, we shall receive:

$$
t_{\max }=\frac{1}{\bar{\beta}} \cdot \ln \left[\frac{\bar{\beta} \exp \left(\bar{\beta} t_{H}\right)-6 \sigma}{\bar{\beta}-6 \sigma}\right]
$$

Let us find the limiting (minimum) value of sensitivity $\mathrm{S}_{\mathrm{e}}$ from equation (14) under the condition of $\beta=0$ and $\mathrm{t}=\mathrm{t}_{\mathrm{vax}}$.

$$
S_{e}=-S_{t}=\frac{\bar{\beta}}{m}=\frac{\bar{\beta}}{m_{0}} \exp (-\bar{\beta} t) .
$$

The relationship between the maximum permissible sensitivity $\mathrm{S}_{\mathrm{e}}$ and the index of instar $\mathrm{k}$ is very tight $(\mathrm{R} 2=0,994)$ and can be described by the exponent:

$$
S_{e}=S_{e, 0} \exp (-\alpha k) \text {, }
$$

where $\mathrm{S}_{\mathrm{e}, 0}=506,84$ day $^{-1} \mathrm{~g}^{-1}$ is the sensitivity of a larva at hatching $(\mathrm{k}=0)$; coefficient $\alpha=1,7004$ demonstrates that the sensitivity of a larva to ecological stress is decreased in $\mathrm{e}=2,72$ times on changing an instar index to the value $\mathrm{k}^{*}=$ $1 / \alpha=0,59$. As it appears, the value $\alpha$ is so huge $\left(\mathrm{k}^{*}<1\right)$ that the sensitivity of a larva from age to age drops more than in e times.

1. An ecological stress effect appears only in the k-th instar. As a result of ecological stress the death rate of larvae in the $\mathrm{k}$-th instar is equal to $\mathrm{H}_{\mathrm{k}}$, and this value will be the same to the end of the fifth instar until the moment of the spinning of the cocoon. The percentage of alive larvae is $\mathrm{W}=100\left(1-\mathrm{H}_{\mathrm{k}}\right)$.

2. An ecological stress effect continues in the next instars. The death rate in each of the instars will be different, and the total is determined on (29) under conditions with $\mathrm{H}_{\mathrm{k}}<1$ for any instar.

$$
W=100 \cdot \prod_{k=k_{S}}^{k=V}\left(1-H_{k}\right)
$$

where $\mathrm{k}_{\mathrm{s}}$ is the number of the instar, in which the ecological stress started. 


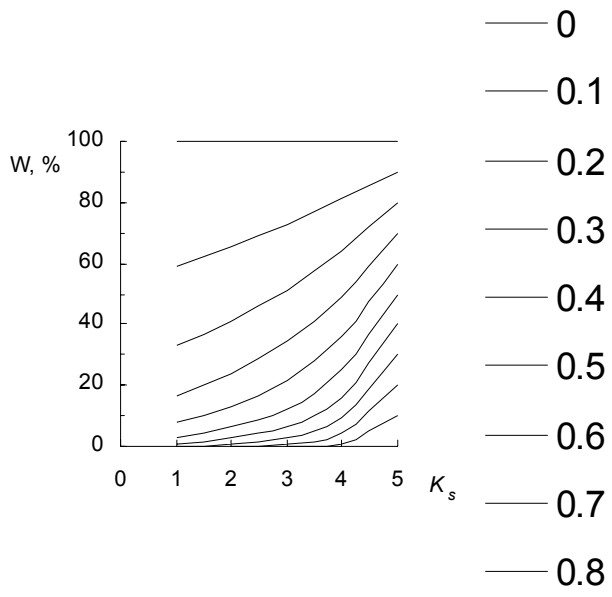

Figure 3: Percentage of live larvae $\mathrm{W}$ until the end of fifth instar.

As shown in the example in Figure 3, we obtained the results of the calculations of the percentage of live larvae $\mathrm{W}$ until the moment of the spinning of the cocoon depending on the number of instar $\mathrm{k}_{\mathrm{s}}$ (the beginning of ecological stress) at the prescribed value of loss $\mathrm{H}_{\mathrm{k}}$ as a parameter.

\section{Conclusions}

1. The created mathematical model of the silkworm's larvae life under the conditions of an ecological stress successfully described a real process. On the basis of numerical calculations the main specifications of the model for different instars of larva are determined. The changes in the accumulation of the larvae's weight in regions with different pollution levels were detected. The method of calculating the death rate and the survival rate of larvae is given depending on the contamination level.

2. On the basis of the obtained model, the experimental results and their analysis it is suggested that the silkworm can be esteemed as a good bioindicator of environmental contamination, first with fluorides and the with other toxic pollutants.

3. As a result, we came to conclusions about the accumulation in the soils, silkworm excrements and the cocoons near the works producing zinc, nickel, mercury, antimony, etc.

4. We have also determined the threshold concentrations of the above mentioned elements in the ecosystem components leading to the mortality of the silkworms and cocoons.

\section{References}

[1] Baars A. J., Van Beek, Spierenburg T. J. et al. Environmental contamination by heavy metals and fluoride in the Saeftinge salt marsh (the Netherlands) and its effect on sheep. Vet. Q, 10, pp. 90-98, 1988. 
[2] Cerklewski F. L., Ridlington J. W. \& Bills N. D. Influence of zinc and iron on dietary fluoride utilization in the rat. J. Nutr., 115, pp. 1162-1167, 1985.

[3] Dabeka R. W. \& McKenzie A. D. Lead, cadmium and fluoride levels in market milk and infant formulas in Canada. J. Assoc. of Anal. Chem., 70, pp. 754-757, 1987.

[4] Davydova V. I. \& Pochashev E. N. The combined effect of manganese and fluorine compounds on the body. Gig. Sanit., 7, pp. 21-25, 1974.

[5] Elsair R. M. J., Denine R., Reggabi M. et al. Boron as a preventive antidote in acute and sub acute fluoride intoxication in rabbits: Its action on fluoride and calcium-phosphorus metabolism. Fluoride, 13, pp. 129 138, 1980.

[6] Leonard, M.D. \& Murray G. T. Sulphonated alizarin fluorine blue: An improved reagent for the positive absorptiometric determination of the fluoride ion. Analyst, 99, pp. 645-651, 1977.

[7] Strehler B. Time, cells, and aging, Academic Press, New York and London, pp.91-101, 1962.

[8] Beeton M., Pearson K., Data for the problem of evolution in man. A first study of the inheritance of longevity, and the selective death rate in man, J. Inst. Actuaries, 35, 1900, pp.112-129.

[9] Putter A., Lebensduaer und Alternsfaktor, Z.Allem.Physiol, 19, 1921, pp.9-36.

[10] Brody S., The kinetics of senescence, J.Gen.Physiology, 6, 1923, pp.245257.

[11] Failla G., The aging process and cancerogenesis. Proc. N.Y. Acad.Sci., 71, 1958,pp.1124-1140.

[12] Failla G., The aging process and somatic mutations, In "The Biology of Aging” (B.L.Strehler et al., eds.), Puzl. No 6, Am.Inst.Biol.Sci., Washington, pp.170-175, 1960.

[13] Simms H.S., Logarithmic increase in mortality as a manifestation of aging, J. Gerontology, 1, (1), 1946, pp.13-26.

[14] Jones H.B., A special consideration of the aging process, disease and life expectancy. Advances in Biol. And Med.Phys., 4, 1956, pp.281-337.

[15] Simms H.S., The use of a measurable cause of death (hemorrhage) for the evaluation of aging, J.Gen.Physiology,26, (2). 1942, pp.169-178.

[16] Sacher G., On statistical nature of mortality with especial reference to chronic radiation. Radiology, 67, 1956, pp.250-257.

[17] Strehler B.L., Mildvan A.S., General theory of mortality and aging, Science 132, (3418), 1960, pp.14-21.

[18] Strehler B.L., Fluctuating energy demands as determinants of the death process (a parsimonious theory of the Gompertz function). In "The Biology of aging" (Strehler B.L. et al., eds.), Publ.No 6, Am.Inst.Biol.Sci., Washington, 1960, pp. 309-314. 